\title{
UNA NUEVA ESPECIE DE TRADESCANTIA (COMMELINACEAE) DEL ESTADO DE QUERÉTARO, MÉXICO
}

\author{
Sergio Zamudio ${ }^{1}$, Adolfo Espejo-Serna ${ }^{2}$, Ana Rosa López-Ferrari ${ }^{2}$ \\ Y JACQUeline CeJA-Romero ${ }^{2}$ \\ ${ }^{1}$ Instituto de Ecología A. C., Centro Regional del Bajío, \\ Apdo. postal 386, 61600 Pátzcuaro, Michoacán, México. \\ ${ }^{2}$ Universidad Autónoma Metropolitana-Iztapalapa, \\ División de Ciencias Biológicas y de la Salud, Departamento de Biología, \\ Apdo. postal 55-535, 09340 México, D.F., México. \\ 2Autor para la correspondencia: sergio.zamudio@inecol.edu.mx
}

\section{RESUMEN}

Se describe e ilustra Tradescantia murilloae Zamudio, Espejo, López-Ferrari et Ceja, una nueva especie del estado de Querétaro. El taxon aquí descrito se compara con $T$. crassifolia Cav., especie con características similares, de la cual difiere por la presencia de un rizoma conspicuo y por las hojas y flores más pequeñas, así como por las características del sustrato y el tipo de vegetación en el que crece.

Palabras clave: Commelinaceae, México, Querétaro, Tradescantia.

\begin{abstract}
Tradescantia murilloae Zamudio, Espejo, López-Ferrari et Ceja, a new species from Querétaro, Mexico is described and illustrated. The new taxon is compared with $T$. crassifolia Cav., a species with similar characteristics from which differs by the presence of a conspicuous rhizome, smaller leaves and flowers and different substrate and vegetation type in which it grows.
\end{abstract}

Key words: Commelinaceae, Mexico, Querétaro, Tradescantia.

En el año 2009 se publicó el fascículo de la Flora del Bajío y de Regiones Adyacentes correspondiente a la familia Commelinaceae (Espejo et al., 2009), en 
el cual se registra la presencia de ocho especies de Tradescantia para la zona de estudio. Sin embargo, como resultado de exploraciones botánicas realizadas recientemente por el primer autor en el municipio de San Joaquín, en el estado de Querétaro, se recolectó material del género que no corresponde a ninguna de las plantas incluidas en el fascículo y que representa además un taxon no descrito para la ciencia, por lo que se propone como:

Tradescantia murilloae Zamudio, Espejo, López-Ferrari \& Ceja, sp. nov. Fig. 1 A y C; Fig. 2 A, C y D.

Herba erecta perennis, usque $60 \mathrm{~cm}$ alta, rhizomatosa, rhizoma breve, plagiotropum; radices cylindrici tuberosi distaliter incrassati; folia ovata vel
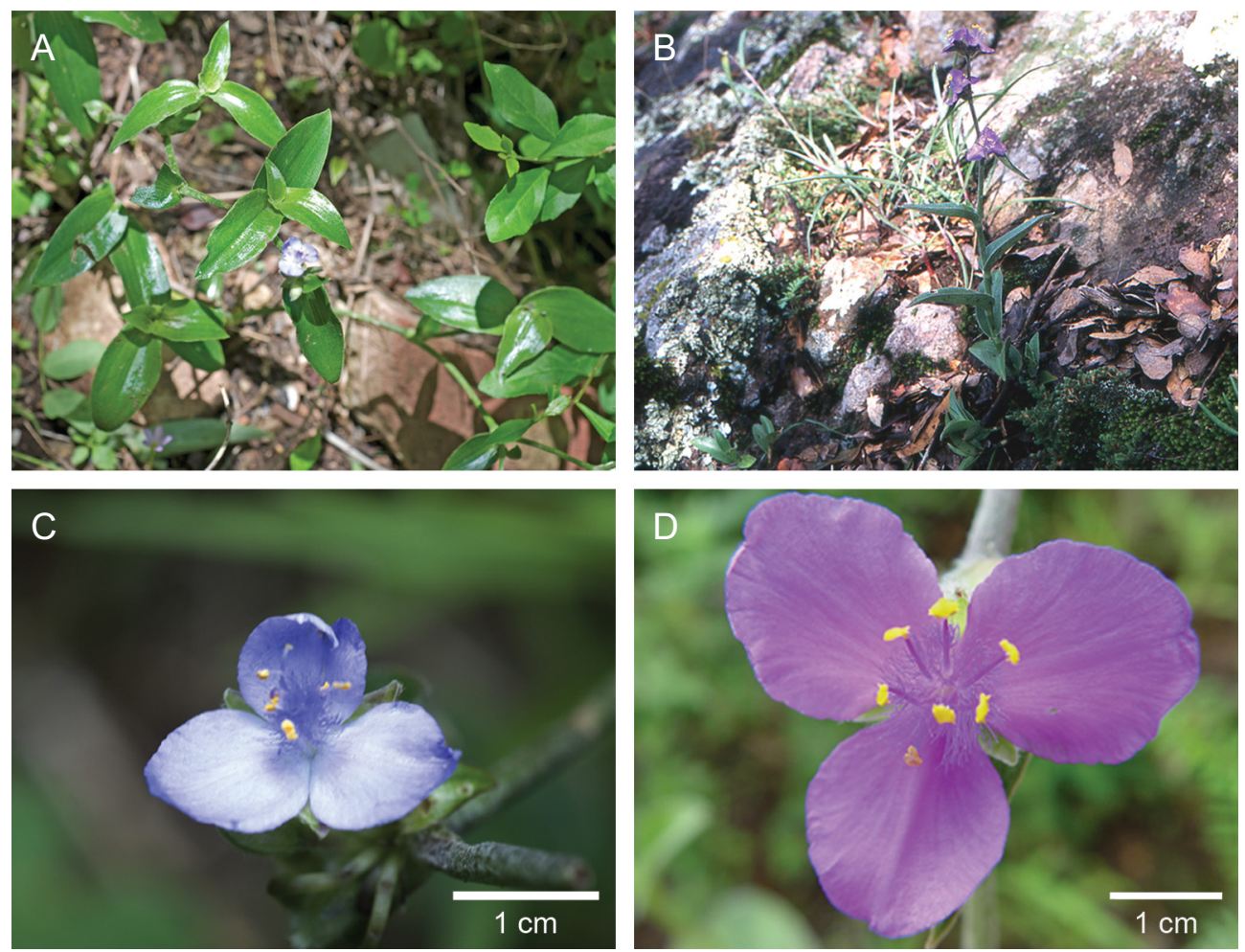

Fig. 1. A. Tradescantia murilloae (S. Zamudio y cols. 15160); B. T. crassifolia (A. R. LópezFerrari y cols. 2494); C. detalle de la flor de T. murilloae (S. Zamudio y cols. 15160); D. detalle de la flor de T. crassifolia (J. Ceja y A. Mendoza R. 1558). 
elliptica, tenua, $1.5-6 \mathrm{~cm}$ longa, $1-2.7 \mathrm{~cm}$ lata, vagina laxa; inflorescentiae plerumque terminales cymis jugatis cum dua folia conjuncta dispositae, pedunculis $5-7.5 \mathrm{~mm}$, acrescentibus et recurvatis post anthesin; flores ca. $2.5 \mathrm{~cm}$ diametro; sepala elliptica vel ovati-elliptica, acuta; petala ample ovata, $12-12.5 \mathrm{~mm}$ longa, $9.5-10.5 \mathrm{~mm}$ lata, acuta, azurea; capsula et semina ignota.

A
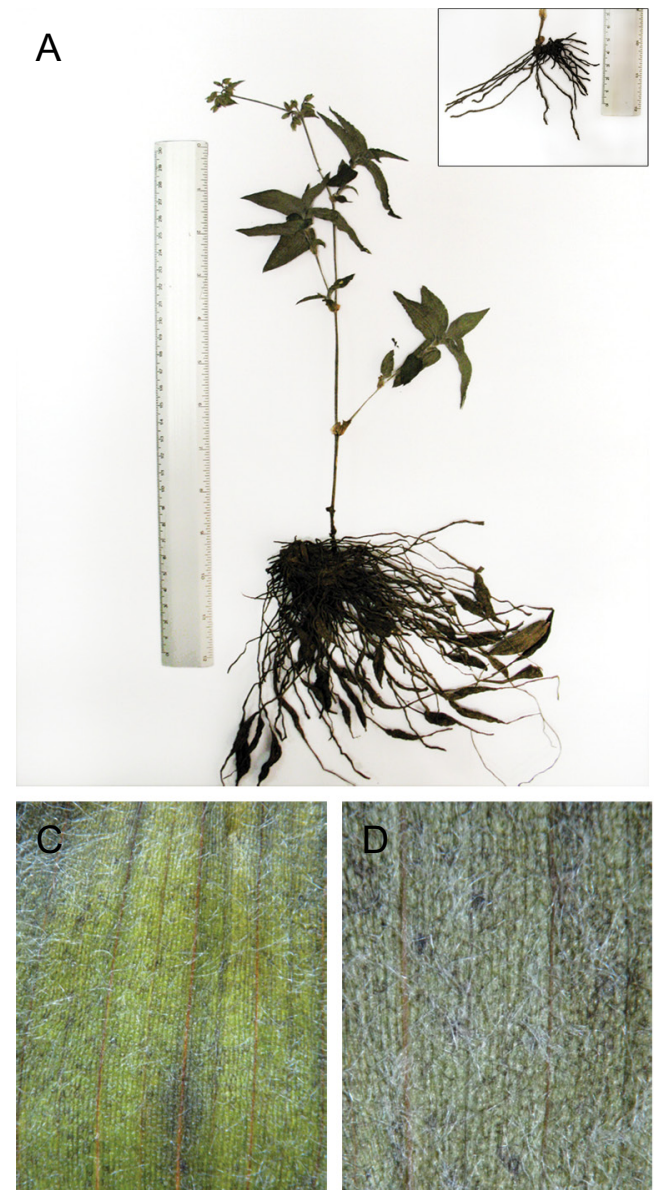
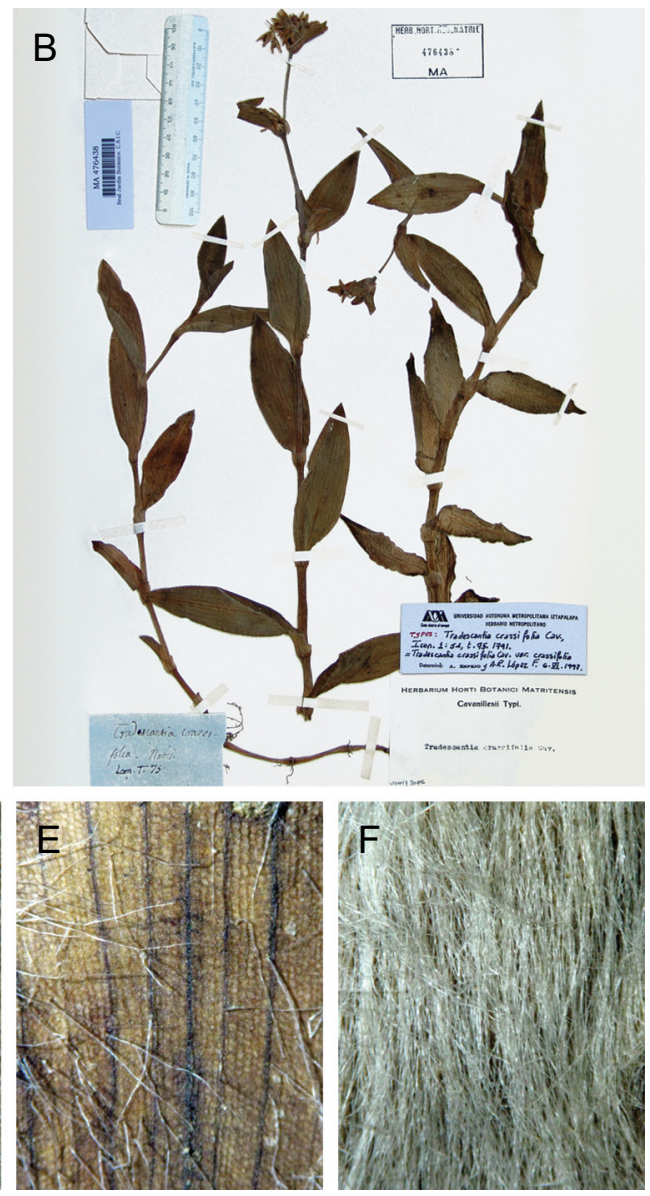

Fig. 2. A. ejemplar completo de T. murilloae (S. Zamudio y cols. 15160); B. material tipo de T. crassifolia en el herbarium cavanillesianum (MA); C. detalle del haz de la lámina foliar de T. murilloae (S. Zamudio y cols. 15160); D. detalle del envés de la lámina foliar de $T$. murilloae (S. Zamudio y cols. 15160); E. detalle del haz de la lámina foliar de T. crassifolia (A. R. López Ferrari y cols. 1207); F. detalle del envés de la lámina foliar de T. crassifolia (A. R. López Ferrari y cols. 1207). 
Planta herbácea perenne de hasta $60 \mathrm{~cm}$ de alto, densamente viloso-lanosa; rizomas cortos, plagiótropos, cilíndricos; raíces cilíndricas, de 15 a $23 \mathrm{~cm}$ de largo, con engrosamientos tuberosos de $2 \mathrm{a} 4 \mathrm{~cm}$ de largo y de ca. $1.5 \mathrm{~cm}$ de diámetro en su porción terminal; tallo cilíndrico, más o menos ramificado pero dejando siempre una porción basal libre de 5 a $11 \mathrm{~cm}$ de largo, densamente viloso-lanoso; hojas ovadas a elípticas, delgadas, de 1.5 a $6 \mathrm{~cm}$ de largo, de 1 a $2.7 \mathrm{~cm}$ de ancho, redondeadas en la base y con una vaina laxa, de 3 a $5 \mathrm{~mm}$ de largo, acuminadas en el ápice, densamente lanoso-vilosas en ambas superficies. Inflorescencias generalmente terminales, formadas por pares de cimas asociadas a dos hojas pareadas, conduplicadas; pedicelos de 5 a $7.5 \mathrm{~mm}$ de largo, viloso-lanosos, acrescentes y recurvados después de la antesis; flores de ca. $2.5 \mathrm{~cm}$ de diámetro; sépalos elípticos a ovado-elípticos, libres, de 6 a $7 \mathrm{~mm}$ de largo, de $3.5 \mathrm{~mm}$ de ancho, verdes, agudos en el ápice, densamente viloso-lanosos; pétalos ampliamente ovados, libres, de 12 a $12.5 \mathrm{~mm}$ de largo, de 9.5 a $10.5 \mathrm{~mm}$ de ancho, azules, agudos en el ápice; estambres subiguales, filamentos lineares, de 5.5 a $6 \mathrm{~mm}$ de largo, azules, densamente barbados cerca de la base, anteras de ca. $1 \mathrm{~mm}$ de largo, amarillas, con el conectivo amplio; ovario globoso, de ca. $2 \mathrm{~mm}$ de largo, de ca. $1.5 \mathrm{~mm}$ de diámetro, densamente piloso, estilo de ca. $4.5 \mathrm{~mm}$ de largo, blanco-azuloso. Cápsula y semillas no vistas.

Tipo: México, Querétaro, municipio de San Joaquín, aproximadamente $5 \mathrm{~km}$ al NE de Apartadero, por la brecha a Álamos, 2059'15.1" N, 99²8'38.1" W, laderas de rocas calizas y lutitas con matorral submontano, $1400 \mathrm{~m}$, 23.VII.2011, S. Zamudio, A. E. Zamudio y R. M. Murillo 15160 (holotipo: IEB; isotipo UAMIZ).

Ejemplares adicionales examinados: Querétaro: municipio San Joaquín, La Redonda, aproximadamente $6 \mathrm{~km}$ al NE de El Apartadero, $21^{\circ} 00^{\prime} 00^{\prime \prime} \mathrm{N}, 99^{\circ} 28^{\prime 2} 23^{\prime \prime}$ $\mathrm{W}$, matorral submontano sobre laderas de rocas calizas, 30.V.2009, S. Zamudio y A. González 14461 (IEB); municipio de San Joaquín, aproximadamente $5 \mathrm{~km}$ al NE de El Apartadero, por la brecha a Álamos, 2059'15" N, 99²8'37" W, 1480 m, laderas calizas con matorral submontano, 20.VII.2011, S. Zamudio y G. Aguilar 15097 (IEB).

Distribución y hábitat: Tradescantia murilloae se conoce hasta ahora sólo del municipio de San Joaquín, en el estado de Querétaro, donde crece sobre laderas con sustratos calizos y vegetación de matorral submontano, entre 1400 y 1480 m s.n.m. Florece durante el mes de julio. 
Etimología: El epíteto específico honra a la Biól. Rosa María Murillo, encargada de la producción editorial de Acta Botanica Mexicana, quien por muchos años ha dedicado su tiempo y su trabajo a esta importante revista.

La nueva especie presenta similitudes con Tradescantia crassifolia, sin embargo difiere de esta especie en varios caracteres, como puede apreciarse en el Cuadro $1 \mathrm{y}$ las figuras anexas.

Cuadro 1. Caracteres comparativos de Tradescantia murilloae Zamudio, Espejo, LópezFerrari \& Ceja y T. crassifolia Cav.

\begin{tabular}{|c|c|c|}
\hline & Tradescantia murilloae & Tradescantia crassifolia \\
\hline Raíces & $\begin{array}{l}\text { Cilíndricas y largas, con } \\
\text { engrosamientos tuberosos alejados } \\
\text { de la base de la planta }\end{array}$ & $\begin{array}{l}\text { Tuberosas, generalmente con los } \\
\text { engrosamientos cercanos a la } \\
\text { base de la planta, ocasionalmente } \\
\text { distales }\end{array}$ \\
\hline Rizoma & Presente & $\begin{array}{l}\text { Ausente, a veces la parte basal del } \\
\text { tallo decumbente y enraizando en } \\
\text { los nudos }\end{array}$ \\
\hline Tallo & $\begin{array}{l}\text { Ramificado por arriba de una } \\
\text { porción basal de } 5 \text { a } 11 \mathrm{~cm} \text { de } \\
\text { largo }\end{array}$ & Simple o poco ramificado \\
\hline Hojas & $\begin{array}{l}\text { Delgadas, ovadas a elípticas, } \\
\text { densa y persistentemente viloso- } \\
\text { lanosas en ambas superficies }\end{array}$ & $\begin{array}{l}\text { Carnosas, oblongo-ovadas } \\
\text { a elípticas o lanceoladas, } \\
\text { glabrescentes en el haz, } \\
\text { densamente lanosas en el envés }\end{array}$ \\
\hline Inflorescencias & Mayormente terminales & Axilares y terminales \\
\hline Pétalos & $\begin{array}{l}12 \times 10 \mathrm{~mm} \text {, azules y agudos en } \\
\text { el ápice }\end{array}$ & $\begin{array}{l}\text { 18-20 x } 17-19 \mathrm{~mm} \text {, rosados a } \\
\text { azul-violáceos y redondeados en } \\
\text { el ápice }\end{array}$ \\
\hline $\begin{array}{l}\text { Estambres } \\
\text { Hábitat }\end{array}$ & $\begin{array}{l}\text { Filamentos } 5.5 \text { a } 6 \mathrm{~mm} \text { largo } \\
\text { matorral submontano, sobre } \\
\text { sustratos calizos }\end{array}$ & $\begin{array}{l}\text { Filamentos } 9 \text { a } 11 \mathrm{~mm} \text { largo } \\
\text { pastizales y sitios abiertos en } \\
\text { bosque tropical caducifolio, } \\
\text { encinares, pinares y matorrales, } \\
\text { sobre diversos sustratos }\end{array}$ \\
\hline
\end{tabular}




\section{LITERATURA CITADA}

Espejo Serna, A., A. R. López-Ferrari y J. Ceja Romero. 2009. Commelinaceae. In: Rzedowski y Calderón de Rzedowski (eds.). Flora del Bajío y de Regiones Adyacentes Fascículo 162. Instituto de Ecología, A. C., Centro Regional del Bajío. Pátzcuaro, México. 122 pp. 